This item was submitted to Loughborough's Research Repository by the author.

Items in Figshare are protected by copyright, with all rights reserved, unless otherwise indicated.

\title{
Aerodynamic drag reduction of a simplified squareback vehicle using steady blowing
}

PLEASE CITE THE PUBLISHED VERSION

http://dx.doi.org/10.1007/s00348-012-1306-4

PUBLISHER

(c) Springer Verlag

VERSION

AM (Accepted Manuscript)

LICENCE

CC BY-NC-ND 4.0

\section{REPOSITORY RECORD}

Littlewood, Rob, and Martin A. Passmore. 2012. "Aerodynamic Drag Reduction of a Simplified Squareback Vehicle Using Steady Blowing”. figshare. https://hdl.handle.net/2134/9851. 
This item was submitted to Loughborough's Institutional Repository (https://dspace.lboro.ac.uk/) by the author and is made available under the following Creative Commons Licence conditions.

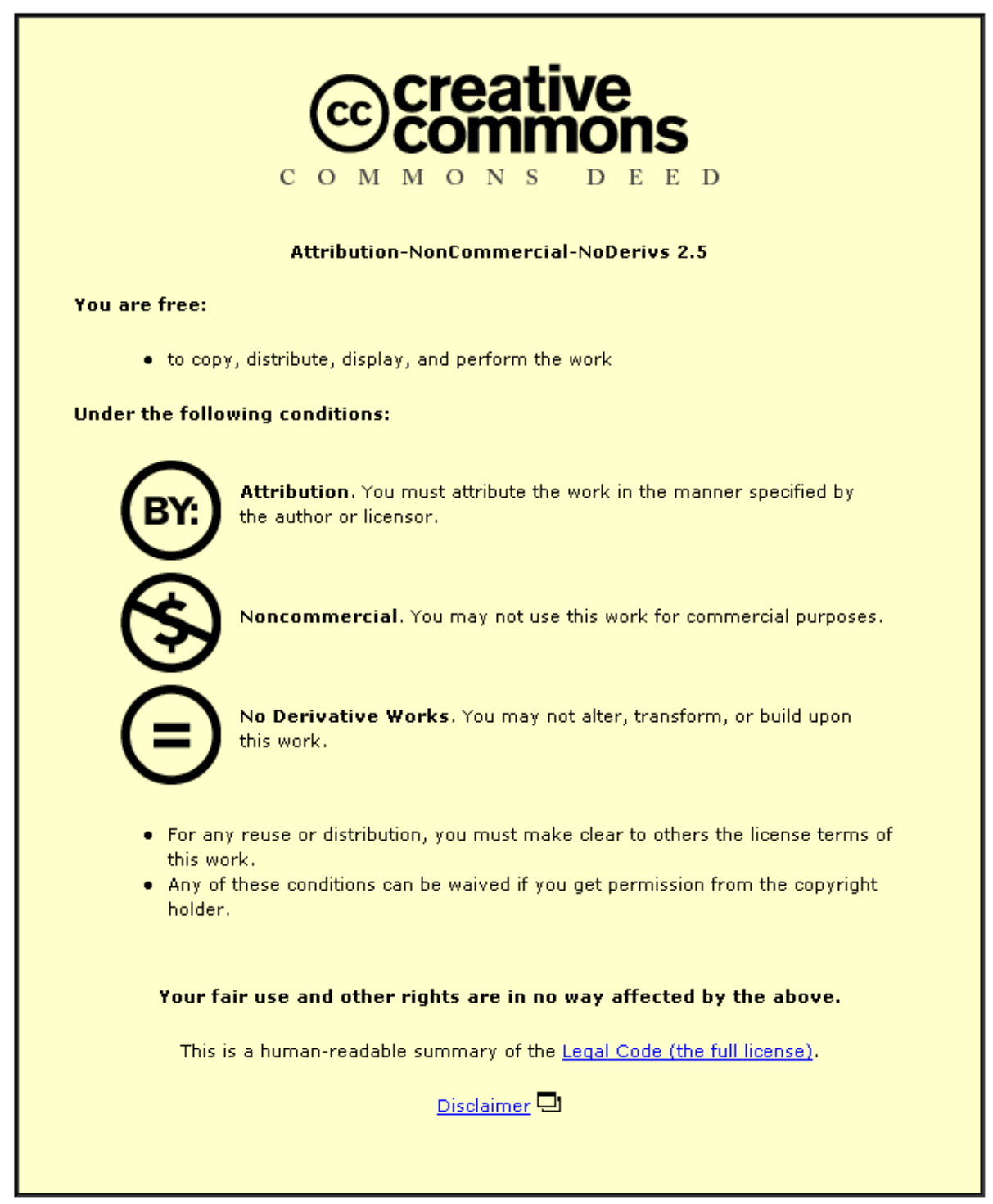

For the full text of this licence, please go to: http://creativecommons.org/licenses/by-nc-nd/2.5/ 


\title{
TITLE:
}

Aerodynamic drag reduction of a simplified squareback vehicle using steady blowing

\section{MAIN AUTHOR:}

\author{
Name: $\quad$ R.P.Littlewood \\ Affiliation: $\quad$ LaVisionUK ltd \\ Downsview House
}

Grove Technology Park

Grove

Oxon

OX12 9FF

United Kingdom

Contact Info: $\quad$ rlittlewood@lavision.com

+44 (0)7917567785

\section{CO-AUTHOR:}

$\begin{array}{ll}\text { Name: } & \text { M.A.Passmore } \\ \text { Affiliation: } & \text { Loughborough University } \\ & \text { Dept. Of Aeronautical and Automotive Engineerin } \\ & \text { Loughborough University } \\ & \text { Loughborough } \\ & \text { Leics } \\ & \text { LE11 3TU }\end{array}$

Contact Info: $\quad$ M.A.Passmore@lboro.ac.uk 


\section{NOMENCLATURE}

\begin{tabular}{|c|c|}
\hline $\mathrm{C}_{\mathrm{d}}$ & Aerodynamic drag coefficient \\
\hline $\mathrm{C}_{\mu}$ & Momentum coefficient \\
\hline 1 & Measured length (mm) \\
\hline $\mathrm{w}$ & Measured width (mm) \\
\hline $\mathrm{h}$ & Measured height $(\mathrm{mm})$ \\
\hline $\mathrm{L}$ & Model length (mm) \\
\hline $\mathrm{W}$ & Model width (mm) \\
\hline $\mathrm{H}$ & Model height $(\mathrm{mm})$ \\
\hline $1 / \mathrm{L}$ & Non dimensional length \\
\hline $\mathrm{w} / \mathrm{W}$ & Non dimensional width \\
\hline $\mathrm{h} / \mathrm{H}$ & Non dimensional ride height \\
\hline$m_{i}$ & Mass flow rate exiting jet $(\mathrm{kg} / \mathrm{s})$ \\
\hline $\mathrm{V}_{\mathrm{i}}$ & Mean jet exit velocity $(\mathrm{m} / \mathrm{s})$ \\
\hline $\mathrm{Q}$ & Freestream dynamic pressure $(\mathrm{Pa})$ \\
\hline A & Model frontal area $\left(\mathrm{m}^{2}\right)$ \\
\hline E & $\begin{array}{l}\text { Ratio of model frontal area to wind tunnel } \\
\text { cross sectional area }\end{array}$ \\
\hline$\Delta \mathrm{t}$ & PIV inter-frame time \\
\hline $\mathrm{L}$ & Model length (m) \\
\hline $\operatorname{Re}$ & Reynolds number \\
\hline Cp & Coefficient of pressure \\
\hline$\overline{\mathbf{C}}_{\mathrm{p}}$ & Area weighted pressure coefficient \\
\hline $1 / \mathrm{L}$ & Non dimensional length \\
\hline $\mathrm{AP}_{\mathrm{NET}}$ & Net change in aerodynamic power (Watts) \\
\hline$\Delta \mathrm{F}_{\mathrm{D}}$ & Change in drag force $(\mathrm{N})$ \\
\hline $\mathrm{V}$ & Freestream velocity $(\mathrm{m} / \mathrm{s})$ \\
\hline$\eta_{i}$ & Blowing system efficiency \\
\hline
\end{tabular}

\section{ABSTRACT}

A large contribution to the aerodynamic drag of a vehicle arises from the failure to fully recover pressure in the wake region, especially on square-back configurations. A degree of base pressure recovery can be achieved through careful shape optimisation, but the freedom of an automotive aerodynamicist to implement significant shape changes is limited by a variety of additional factors such styling, ergonomics and loading capacity. Active flow control technologies present the potential to create flow field modifications without the need for external shape changes and have received much attention in previous years within the aeronautical industry, and more recently, within the automotive industry.

In this work the influence of steady blowing applied at a variety of angles on the roof trailing edge of a simplified $1 / 4$ scale square-back style vehicle has been investigated. Hot wire anemometry, force balance measurements, surface pressure measurements and PIV have been used to investigate the effects of the steady blowing on the vehicle wake structures and the resulting body forces.
The energy consumption of the steady jet is calculated and is used to deduce an aerodynamic drag power change. Results show that overall gains can be achieved, however the large mass flow rate required restricts the applicability of the technique to road vehicles. Means by which the mass flow rate requirements of the jet may be reduced are discussed and suggestions for further work put forward.

\section{INTRODUCTION}

It is clear that the reduction of $\mathrm{CO}_{2}$ emissions from road vehicles is currently, and will continue to be a significant area of research for the automotive industry.

The effect on $\mathrm{CO}_{2}$ emissions from fossil fuelled vehicles as a result of reduced aerodynamic drag forces can be significant, but is largely dependent on what drive cycle is used in this calculation (Horn 201;1Schultz 2010), however previous work has shown that a saving of $10 \%$ in aerodynamic drag can result in a $3 \%$ saving in fuel consumption (Sovran 1983). The effect of aerodynamic drag also becomes important when considering vehicle range requirements for low energy density power sources such as batteries and fuel cells.

It is worthy of note that whilst aerodynamic drag coefficients $\left(\mathrm{C}_{\mathrm{d}}\right)$ have fallen significantly over the past thirty years, vehicle frontal areas have seen an increase (Hucho 1998). This inverse tendency illustrates the difficult trade-offs faced by vehicle manufacturers when considering consumer requirements and buying trends, but it is apparent that vehicle styling is still a dominant factor when creating vehicle body shapes.

For this reason the vehicle aerodynamicist must use ever more discrete means of achieving drag reductions, such as wheel spoilers (Ribaldone 2010), underbody smoothing and sealing of under bonnet gaps (Schrefl 2010). In recent years active flow control techniques have been of research interest due to their potential ability to achieve flow modification and drag reductions without the need for physical shape changes (Glezer 2002), however they have yet to be proven in a real world vehicle application.

In fundamental studies of active flow control techniques the techniques are generally implemented at, or near, points of separation in order that the formation of the wake may be controlled (Lin 1999; Brunn 2006). Generally in 2D bluff body applications periodic structures that are normally shed into the body wake are targeted by the control technique in the hope that wake size can be modified.

Often the goal of the investigations is to reduce the size of the wake (Roumeas 2008) in order to reduce drag, 
however in certain applications it can be argued that a stretched wake structure would create lower drag (Bearman 1983; Pastoor 2008), and no general rule for every bluff body can be applied. However for the bluff body model used in the work reported here a reduction in wake size has been shown to result in a reduction in drag force (Littlewood 2010).

Previous studies by Englar (2003) and Geropp (2000) used a trailing edge radius in combination with steady blowing at the rear of squareback vehicles to create a coanda effect. This was used to turn freestream flow towards the centre of the wake region to increase the pressure on the base of the vehicle. The requirement for large trailing edge radii in this technique means that when blowing is not in operation a drag penalty is present due to the suction peaks created around the trailing edge radii. In road vehicle optimisation large trailing edge radii are often avoided in order to negate these suction effects and sharp trailing edges that result in steady, distinct separation locations are desirable. Additionally such a large trailing edge radius would not normally be acceptable to a vehicle stylist.

Rouméas et al (2008) performed CFD studies using a sharp edged squareback vehicle and steady blowing oriented at various angles relative to the freestream direction. It was concluded that a jet angled into the wake at 45 degrees created a turning of the flow and a base pressure increase. The current work investigates this effect experimentally using a $1 / 4$ scale simplified squareback vehicle model, fitted with a pressurised cavity and variable geometry jet outlet on the roof trailing edge. Investigations have already been performed on the same model using a small passive optimisation to the rear roof trailing edge which is used as a bench mark (Littlewood 2010).

\section{EXPERIMENTAL SETUP AND DATA ACQUISITION}

\section{Wind tunnel}

All tests were performed in the Loughborough University closed working section, open circuit wind tunnel. The working section of the tunnel is $1.92 \mathrm{mx} 1.32 \mathrm{~m}$ which gives a blockage of $4.4 \%$ for the current model. Turbulence intensity in the working section is under $0.2 \%$, and the working section floor is stationary. Force measurements were made using a six component under-floor balance that was coupled to the model using four $8 \mathrm{~mm}$ diameter pins. The ride heights chosen for the tests were 30,40 and $70 \mathrm{~mm}$ giving a non-dimensional ride height of $10.3 \%, 13.8 \%$ and $24.1 \%$ based on the model height. A Reynolds sweep was performed and established that the model was insensitive to Reynolds number above approximately 1.4 million based on the model length. The subsequent tests were performed with free stream velocities of 25 ,
30,35 and $40 \mathrm{~m} / \mathrm{s}$, equating to length based Reynolds numbers of 1.94, 2.32, 2.71 and 2.9 million. Multiple freestream velocities were tested to ensure Reynolds number dependent effects were not created when blowing was applied but also as a means of creating a wider range of values of jet momentum coefficient $\left(\mathrm{C}_{\mu}\right)$ in addition to making changes to jet exit velocities.

\section{Model}

The Windsor model, used in this work is shown in Fig. 1 and Fig. 2. All the leading edges are well rounded to avoid separation with radii of $0.05 \mathrm{~m}$, with the exception of the roof leading edge which has a radius of $0.20 \mathrm{~m}$. The longitudinal and rear edges are sharp. A NACA0021 wing profile with $150 \mathrm{~mm}$ chord protrudes from the centre of the model floor, to below the wind tunnel floor. This wing is fitted as shielding for pneumatic tubing and cabling that needs to be routed inside the model from underneath the working section. Four flexible tubes connect a machined cavity inside the model (Fig. 1) to a pressure regulator and compressed air supply. Measurements of cavity pressure are taken via four pressure tappings in the cavity connected to a Furness Controls manometer with an accuracy of \pm 2.5 $\mathrm{mmH} 2 \mathrm{O}$. All cables and pneumatic tubes are hung vertically downwards from the model for a minimum length of 1 metre before being coupled to their corresponding connections to minimise grounding effects on the balance measurements of the drag forces.

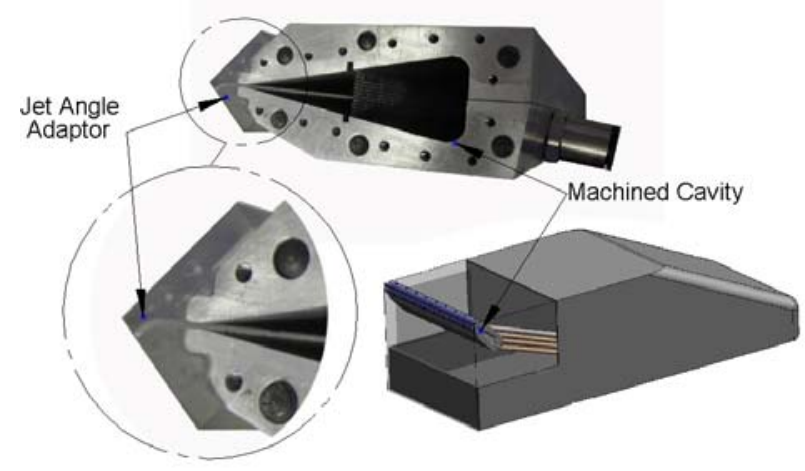

Fig. 1 Windsor model showing pressurised cavity and jet angle adaptor component

The angle at which the jet exits the model is modified using removable 'jet angle adaptors' (Fig. 1) manufactured from SLA resin, which also form the roof trailing edge of the model. The jet angle adaptors maintain a sharp 90 degree roof trailing edge to ensure the model configuration stays as a squareback, whilst the jet exit angle is modified. The jets exit configurations tested were 'vertically up', 'angled upwards and downstream', 'horizontally downstream', and 'angled downwards and downstream' and will be referred to as configurations A, B, C, and D respectively (Fig. 2). 


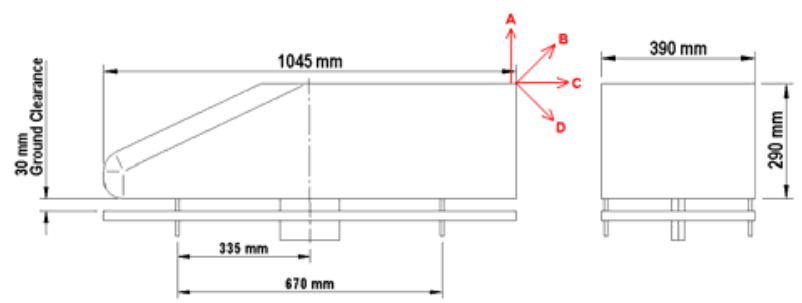

Fig. 2 Model dimensions and geometric jet exit configurations

The jet exit spans the full width of the model but is divided into nine equal sections by $0.5 \mathrm{~mm}$ wide supports. The divisions are required to prevent the slot width increasing when air pressure is applied. The slot width is $1 \mathrm{~mm} \pm 0.05 \mathrm{~mm}$.

\section{Slot exit velocity measurements}

In order to quantify the jet exit velocity a single wire hot-wire anemometer was positioned $1 \mathrm{~mm}$ above the jet outlet, oriented in line with the jet exit axis. The hotwire was calibrated between $0-60 \mathrm{~m} / \mathrm{s}$ using a Dantec hotwire calibrator. At each location 5000 samples were acquired at a frequency of $1000 \mathrm{~Hz}$. The signal was hardware filtered using a low pass filter at $300 \mathrm{~Hz}$. The probe was traversed along the width of the model in $1 \mathrm{~mm}$ steps to generate an average jet exit velocity. The average was used in the calculation of a jet momentum coefficient using Equation 1, where $\dot{m}$ is the jet mass flow rate, $V_{j}$ is the mean jet exit velocity, $q$ is the freestream dynamic pressure and $\mathrm{A}$ is the model frontal area. This momentum coefficient non-dimensionalises the momentum of the air blown from the jet against the air in the freestream.

$$
\text { Equation } 1 \quad C_{\mu}=\frac{\dot{m}_{j} v_{j}}{q A}
$$

The presence of a $\mathrm{V}^{2}$ term in the numerator for the jet and the denominator for the freestream flow means that manipulation of the relationship between the jet exit velocity and the freestream velocity creates more significant changes in $\mathrm{C}_{\mu}$ than a manipulation of the physical size of the model to slot width ratio. Previous authors (Lin 1999) have placed an emphasis on the requirement for high $\mathrm{C}_{\mu}$. In the current work higher $\mathrm{C}_{\mu}$ values could be achieved using lower freestream velocities, however freestream velocities were only reduced within a region where results were found to be insensitive to Reynolds number.

The presence of a $\mathrm{V}^{2}$ term also creates additional factors to consider when deciding how best to scale the technology to full scale. An optimum choice of jet outlet width and jet outlet velocity for the minimum mass flow rate requirement and power input would give the optimum choice of $\mathrm{C}_{\mu}$.

\section{Force measurements}

The model was mounted on a six component underfloor balance. All force measurements were acquired for 30 seconds at a data acquisition rate of $5 \mathrm{~Hz}$ block averaged from the raw voltage signal running at $4.6 \mathrm{KHz}$. Using an example set of data acquired at 40 $\mathrm{m} / \mathrm{s}$ freestream velocity Fig. 3 shows that measured drag coefficients recorded in a 30 second sample are accurate to better than $\pm 0.001 \mathrm{Cd}$ ( 1 count) with $99 \%$ confidence.

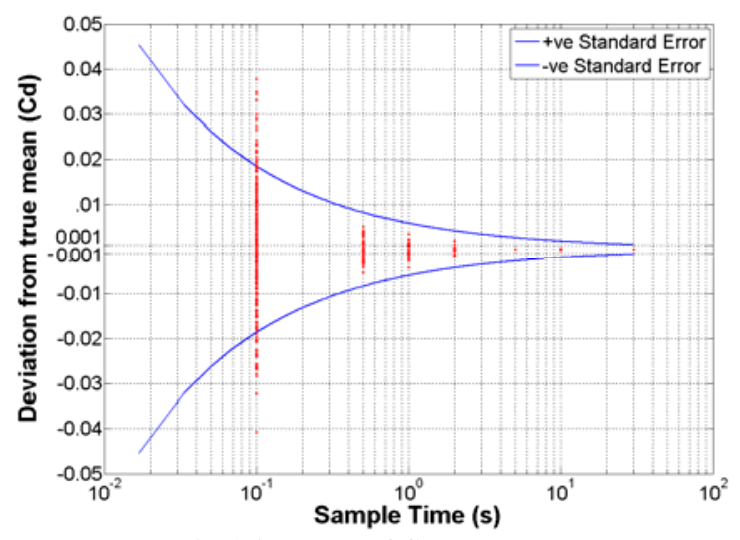

Fig. 3 Accuracy of Cd measurements

Measurements of baseline drag coefficient were repeated 18 times over a period of 3 days. During the 18 baseline tests the model was removed and reinstalled on the balance twice, and measurements were taken with and without all tubing and cables connected. Within these repeats the fluctuation in measured drag coefficient for the model never exceeded 2 counts $(0.002 \mathrm{Cd})$, indicating the overall repeatability and that the installation of the tubing had little influence on the measured balance data.

The baseline thrust force produced by each jet configuration was measured in quiescent conditions and subtracted from measured drag forces on the model when in freestream flow with the jets active.

Measured $\mathrm{C}_{\mathrm{d}}$ values were corrected for blockage using the MIRA area ratio method shown in Equation 2, where $\mathrm{E}$ is the ratio of model frontal area to working section cross sectional area.

Equation 2

$$
C_{\text {Fcorrected }}=\frac{C_{\text {Fmeasured }}}{(1+2 E)}
$$

\section{Pressure measurements}

The base of the model was fitted with 111 surface pressure tappings which were connected via flexible tubing to two 64 channel pressure scanners mounted inside the model. Data was acquired from the scanners via CAN technology which required two Ethernet cables to be routed through the wind tunnel floor and through the model floor at its centre-line. The worst 
case static accuracy of the scanners ranged between $\pm 0.06 \%$ to $\pm 0.1 \%$ as a percentage of full scale $( \pm 232 \mathrm{mmH} 2 \mathrm{O})$ depending on operating conditions. All pressure coefficient data was corrected for blockage using Equation 3.

$$
\text { Equation } 3 \quad C_{\text {Pcorrected }}=\frac{C_{\text {Pmeasured }}+2 E}{(1+2 E)}
$$

\section{PIV}

A LaVision dual camera 2D planar PIV system using a dual pulsed Nd:YAG laser to produce a light sheet in the stream-wise plane along the centre line of the model was set up as shown in Fig. 4. Each camera has $2048 \mathrm{x}$ 2048 pixels, and the inter-frame time $(\Delta t)$ for image pairs was tuned in order to generate mean particle displacements of 6-8 pixels. Image pairs were acquired at $5 \mathrm{~Hz}$ and a sample of 1000 image pairs (Hollis 2004) was taken for each configuration tested. A sliding average of the minimum background light intensity in three consecutive image pairs was calculated for each set of 1000 images. This sequence of background light intensity was then subtracted from each image pair in sequence in order to reduce the effects of background light on the correlation process. A multi-pass decreasing window size correlation processing algorithm was used with a final window size of $32 \times 32$ pixels, with a $50 \%$ overlap. This final window size gives a vector grid resolution of $5 \mathrm{~mm} \times 5 \mathrm{~mm}$ over an unmasked field of view of $350 \mathrm{~mm} \times 750 \mathrm{~mm}$.

The location of the seeding rake and the acquisition parameters were tuned until a minimum of $95 \%$ first choice vectors were present in the instantaneous images. Seeding was generated by a laskin nozzle oil seeder located approximately 1.5 model lengths upstream of the model front. Previous experiments have indicated that although the seeder location does have an effect on the absolute measured drag values, trends and changes due to configuration changes remain the same (Littlewood 2010).

The accuracy of mean velocity calculations at any spatial co-ordinate within the measurement plane is a function of the local RMS. Analysis of individual vector locations at positions chosen close to the model base surface and in the freestream were used to generate statements of accuracy from 1000 image pairs. At positions close to the model base mean velocities are accurate to $\pm 0.25 \mathrm{~m} / \mathrm{s}$ to $95 \%$ confidence. The position close to the model base is in a region of high RMS. Other regions of low RMS such as in the freestream produced better accuracies of $\pm 0.12 \mathrm{~m} / \mathrm{s}$ to $95 \%$ confidence.

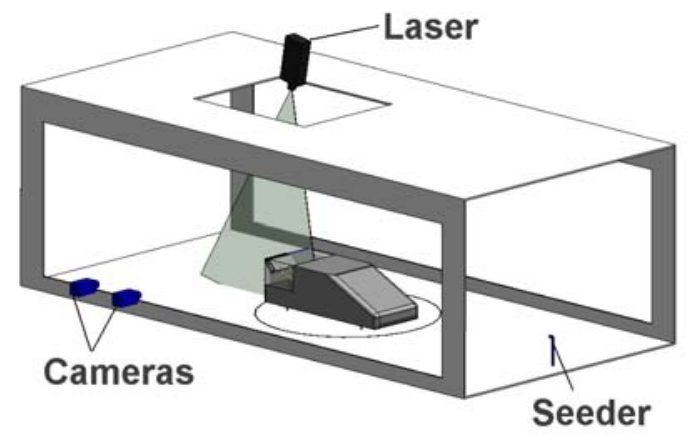

Fig. 4 PIV experimental setup

\section{EXPERIMENTAL RESULTS}

\section{Jet Characterisation}

Fig. 5 shows an example jet exit velocity profile across one half of the model width, and illustrates the need to use an averaged jet exit velocity. The width of the slot incorporates physical divisions for strength, and local minima can be seen where the structural divisions were located to strengthen the slot. The velocity measurements at these locations were removed from the calculation of the mean exit velocity, which was only calculated over the open slot area. These data points were removed because the calculation of $\mathrm{C}_{\mu}$ should only be based on flow exiting the slot outlet and not any velocity created by entrainment of the surrounding medium.

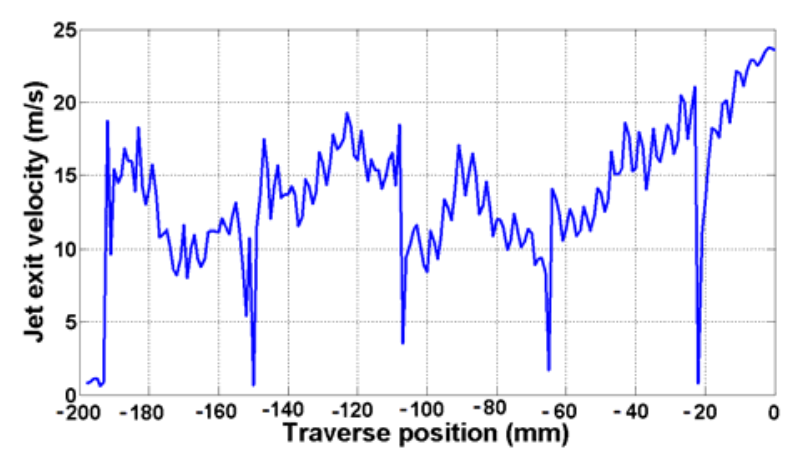

Fig. 5 Example hot wire measurement of jet exit velocity profile

The orientation of jet nozzle exit on the jet angle adaptors $\mathrm{A}, \mathrm{B}, \mathrm{C}$ and $\mathrm{D}$ were manufactured to be A; vertically up B; at 45 degrees between vertically up and horizontally downstream $\mathrm{C}$; horizontally downstream and $\mathrm{C}$; at 45 degrees between vertically downwards and horizontally downstream. In quiescent conditions the measured jet axis of the jet exit orientations for configuration $\mathrm{A}, \mathrm{B}$ and $\mathrm{C}$ were well aligned with the geometric jet exit axis. However in configuration D it was noted that the jet was vectored downwards towards the base wall of the model, This results in a measured jet axis angle of approximately 9.5 degrees. This is shown in Fig. 6 where the jet exit position is at $\mathrm{x}=0 \mathrm{y}=0$, the black line shows the actual jet axis centre, and the red line shows the geometric axis 
at 45 degrees. From this result it is clear that any future testing using a similar arrangement of jets should also quantify the angle that the air leaving the jet exits at relative to the 'geometric' or physical exit angle of the jet outlet/walls.

The change in jet exit angle relative to the geometric outlet angle is introduced in configuration $\mathrm{D}$ because of the need to use internal curved ducts to direct the jet out of the model at a downwards angle. The curved ducts create introduce a coanda effect at the outlet of the jet meaning the air is vectored further downwards.

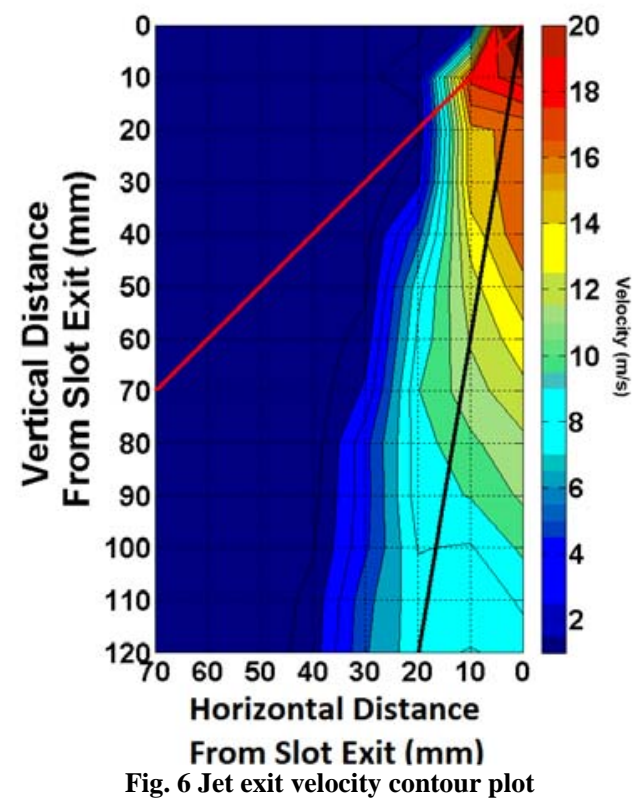

\section{Balance Results}

Of the four configurations tested only configuration D produced a reduction in drag coefficient. Results are therefore examined in more detail for configurations B and D only.

Fig. 7 shows the changes in drag coefficient for configuration $B$ vs. increasing jet momentum coefficient. At all the ride heights tested the drag gradually increased with increasing momentum coefficient and the results showed the same trends at different model length based Reynolds numbers. In configuration $\mathrm{B}$, with the jet acting at 45 degrees upwards, at the point of separation it is suggested that the size of the wake is increased by deflecting the shear layer upwards. Some theories regarding the influence of wake size on resultant body forces predict that a stretched wake will create increased base pressures by moving vortex structures away from the base surface and so reduced body drag forces (Pastoor 2008; Bearman 1983). Other published work (Rounmeas 2008) suggests that a reduction in wake size will increase base pressures and create lower drag forces. Fig. 7 supports the theory that a larger wake will create larger drag forces.
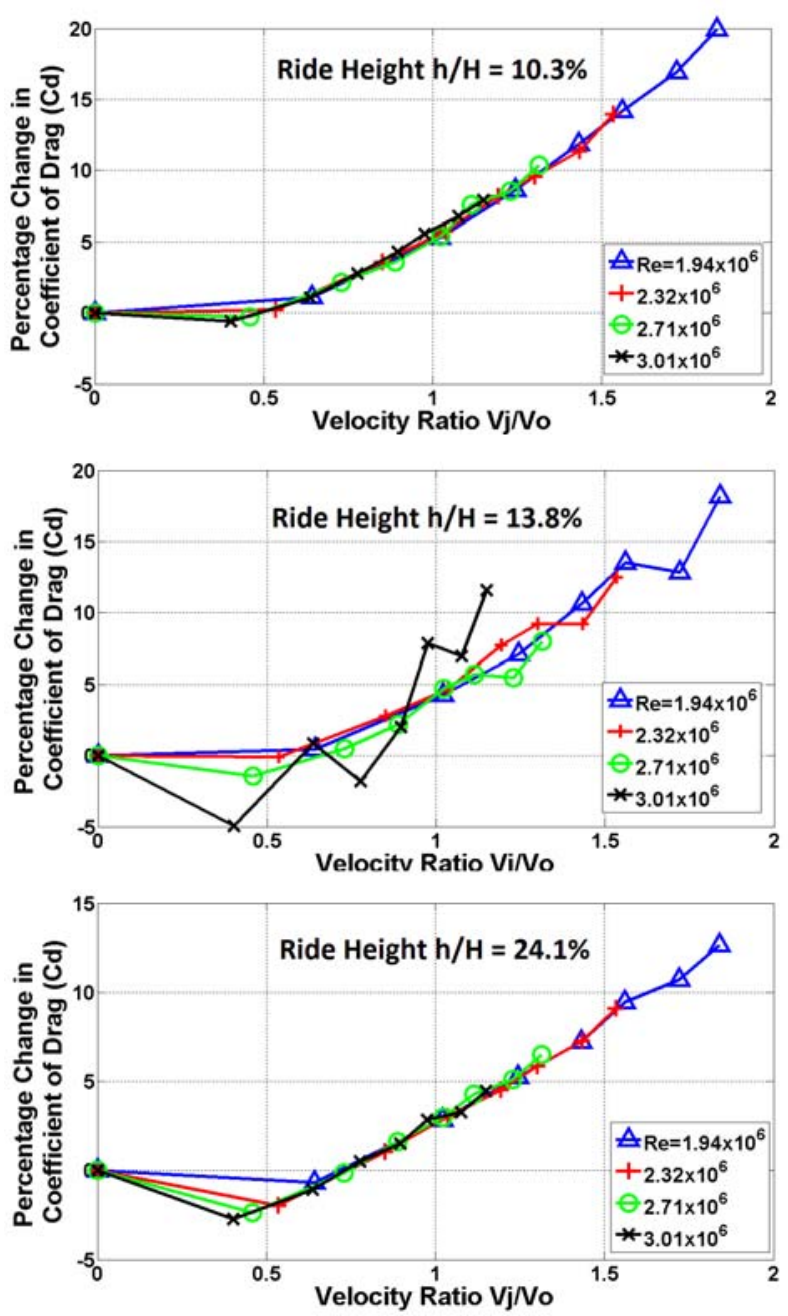

Fig. 7 Changes in balance measured $C_{d}$ for configuration $B$ at ride heights of $10.3 \%, 13.8 \%$ and $24.1 \%$

The absolute change in $C_{d}$ for a given momentum coefficient is increased as the ride height is decreased. If the drag force changes are linked to wake size and it is the dominant factor involved, it would suggest that at lower ride heights the wake size is increased more for a given momentum coefficient. However the change in drag reduction as ride height is reduced from the extreme case of $\mathrm{h} / \mathrm{H}=24.1 \%$ to $\mathrm{h} / \mathrm{H}=10.3 \%$ is an average of 1.7 times greater. If the change in wake size was anything close to proportional to the changes in drag forces then the wake size increase required to create the measured force changes would also be significant. For this reason it is believed that additional factors within the wake have an influence on base pressures and hence body drag forces.

Fig. 8 shows the changes in drag coefficient as a result of blowing using configuration $\mathrm{D}$, at a ride height of $\mathrm{h} / \mathrm{H}=10.3 \%$. Significant reductions in $\mathrm{C}_{\mathrm{d}}$ are observed which gradually increase as momentum coefficient is increased. As seen in other configurations the trends are not sensitive to Reynolds number, which allows larger $\mathrm{C}_{\mu}$ values to be tested by reducing freestream the velocity. 


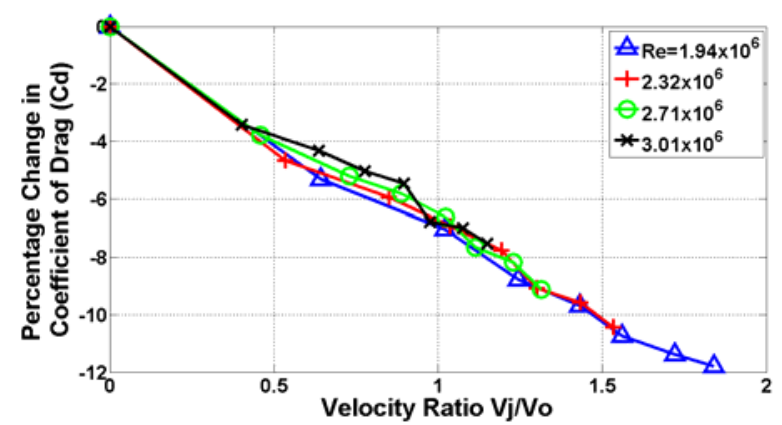

Fig. 8 Changes in balance measured $C_{d}$ for the Configuration $D$ at a ride height of $\mathbf{1 0 . 3 \%}$

As per Fig. 7 it would be reasonable to attribute the drag reductions seen in Fig. 8 to a deflection of the shear layer downwards creating a reduction in the overall wake size. However the drag reductions are so significant $(3 \%-12 \%)$ that if wake size alone is the dominant influence on base pressure and body forces the wake size reduction would be significant. The magnitudes of drag changes observed support the theory that additional 'in wake' factors may have an influence on drag forces in addition to the modification of wake size alone. In order to investigate the changes further, base surface pressures and in wake PIV measurement techniques were used.

In the following sections a test Reynolds number of $3.01 \times 10^{6}$ has been chosen for further analysis. This Reynolds number has been selected as it creates the least favourable $\mathrm{C}_{\mu}$ values tested but also the most representative values if the technology were to be scaled up to production vehicle magnitudes.

\section{Pressure Measurements}

In the baseline configuration the base surface pressures exhibit a similar pattern observed for this configuration in previous work (Littlewood 2010)(Fig. 9). The main feature observed in the base pressure contours is a large region of suction on the lower half of the base surface between $\mathrm{h} / \mathrm{H}=0$ and $\mathrm{h} / \mathrm{H}=0.4$. On the upper half of the base the pressures gradually increase towards the roof trailing edge, and are more uniform across the base width, indicating the two dimensional nature of the flow in this region. Immediately below the roof trailing edge a reduction in pressure is observed where separation occurs. a)

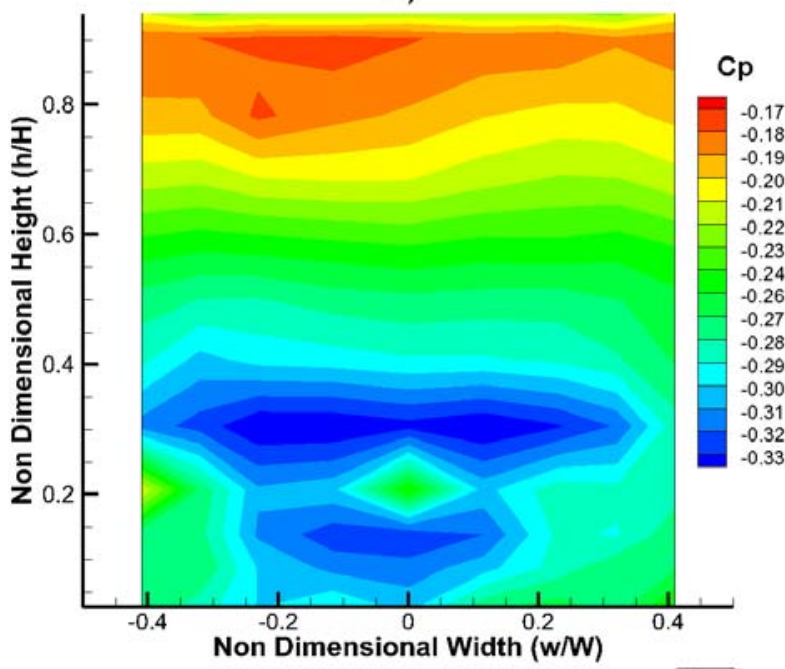

b)

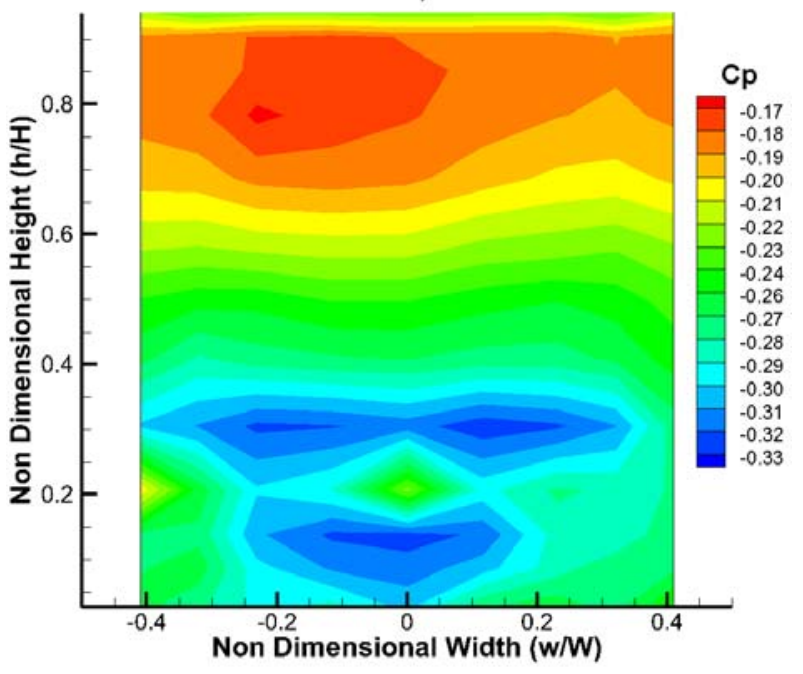

Fig. 9 Base pressure contours for a)baseline and b) blowing configuration $D$ at $C \mu=0.013$ and $\operatorname{Re}=3.01 \times 10^{6}$

When blowing is applied in Configuration D at a momentum coefficient of 0.013 a global increase in base pressures is observed (Fig. 9b). The pressure contour patterns observed in Fig. 9a remain present in Fig. $9 b$ however the low pressures observed in the lower half of the base for the baseline configuration have increased with the application of blowing. This increase has resulted in a more uniform distribution of base pressures and a less pronounced suction region between $\mathrm{h} / \mathrm{H}=0$ and $\mathrm{h} / \mathrm{H}=0.4$.

The area weighted $C_{d}$ changes were calculated using Equation 4 and are compared to those measured by the balance in Fig. 10.

Equation 4

$$
\overline{C_{p}}=\frac{1}{A} \int_{A} C_{p} d A
$$




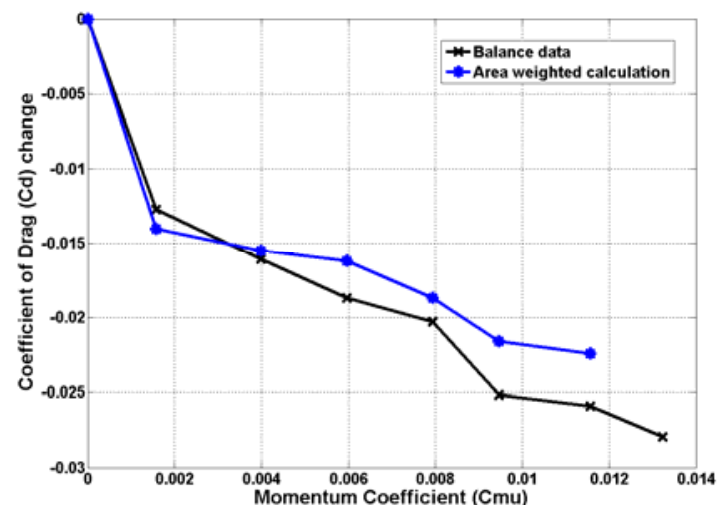

Fig. 10 Balance and area weighted calculations of changes of $C_{d}$ Configuration $D$ at $\operatorname{Re}=3.01 \times 10^{6}$

Fig. 10 illustrates that the changes in total model drag measured by the balance are primarily as a result of changing pressures on the base surface, as opposed to changes in pressures on forward facing surfaces at the front of the model. As momentum coefficient increases the pressure data trends show a good match with the balance data and highlight the significant initial reduction of 13-14 counts when $\mathrm{C}_{\mu}=0.0016$.

\section{PIV}

Fig. 11 shows time averaged PIV vector fields of the baseline un-blown case at a Reynolds number of $3.01 \times 10^{6}$, acquired from the measurement plane shown in Fig. 4. The rear portion of the model is shown as a solid black region and the background contour colours denote vorticity with velocity magnitude vectors in the foreground.

The size of the wake produced by the model is significant as may be expected for a squareback vehicle, and the full thickness of the shear layer is identifiable as the vorticity contour plot magnitudes blend from orange into white. Within the near wake between $L / 1=0$ and $\mathrm{L} / \mathrm{l}=-0.35$ strong structures are seen in close proximity to the base. The dominant structure is a lower recirculating vortex that interacts with the model base surface causing low base pressures as shown in Fig. 9a and documented in Littlewood(2010).

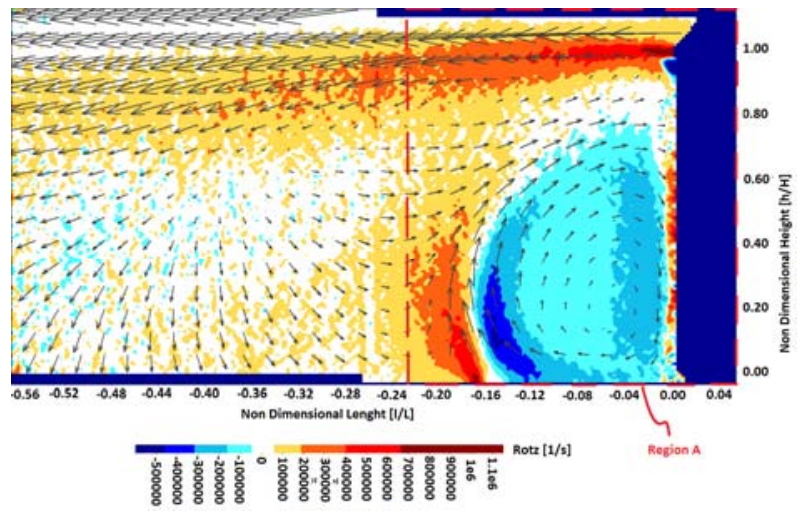

Page $\mid 7$
Fig. 11 Time averaged PIV image for unblown baseline configuration $\mathrm{h} / \mathrm{H}=10.3 \%, \mathrm{Re}=3.01 \times 10^{6}$. Velocity magnitude shown in background with velocity vectors in foreground

Fig. 12 shows the same measurement plane when blowing is applied in the 'downward blowing configuration' (D) at $\mathrm{C}_{\mu}=0.012$. An overall wake size reduction is observed but it is not as significant as expected from the drag reductions observed in Fig. 8. To illustrate the change in wake size a streamline was plotted at the point of separation at the rear trailing edge of the model in the blown and unblown case. This streamline is then used to indicate the the outer edge of the model wake in both cases. Both streamlines are plotted in figure $\mathrm{xx}$ where the upper streamline shows the boundary of the unblown configuration wake, and the lower streamline shows boundary of the blown configuration wake. If these streamline boundary positions are used to calculate a $2 \mathrm{D}$ wake area change between the two configurations then the application of steady blowing creates a $14.2 \%$ wake size reduction for a $7.5 \%$ reduction in drag (Fig. 8 ). When this method is applied to previous work using passive optimisation (Littlewood 2010) a small chamfer that lead to a $4.4 \%$ drag reduction is associated with a $11.1 \%$ wake size reduction.

It is recognised that some $3 \mathrm{~d}$ trailing vortices may be appearing at the upper corners of the roof trailing edge in both the passive optimisation case (Littlewood 2010) and the actively blown case discussed here. Any large scale $3 \mathrm{~d}$ structures would mean that using the centreline PIV plane as an indication of wake size would become invalid. However in Littlewod(2010) base pressure contributions and slant angle contributions were analysed to conclude that the flow structure remains largely 2D across the span of the model base, with the application of small chamfer angles. Additionally, the majority of changes in body drag forces are as a result of base pressure recovery. In the current work, using the same model, no slant angle is present and the application of downward blowing shows no onset of $3 \mathrm{~d}$ vortices at the upper corners. This can be concluded by comparing the base pressure contour plots with and without blowing (Fig. 9), and also comparing these against similar plots in Littlewood(2010) where 3d Cpillar vortices did begin to occur at the larger chamfer angles studied.

As the lower vortex structure is linked to a large region of suction, it may be reasonable to assume that the significant reductions in drag observed in Fig. 8 are a result of modification to this lower structure, and its interaction with the base surface in addition to the global reduction in wake size. In effect, the benefits of stretching the wake to move vortices further away from the base outlined by Bearman (1983) and Pastoor (2008), are, in this instance, created by the downward blowing of the jet weakening the lower vortex structure. The weakening of this structure is shown in the reduction in the magnitude of vorticity of the structure,

Page 
comparing Fig. 11 to Fig. 12. This reduction in vorticity inevitable leads to a reduction in the local velocity interacting with the model base surface.

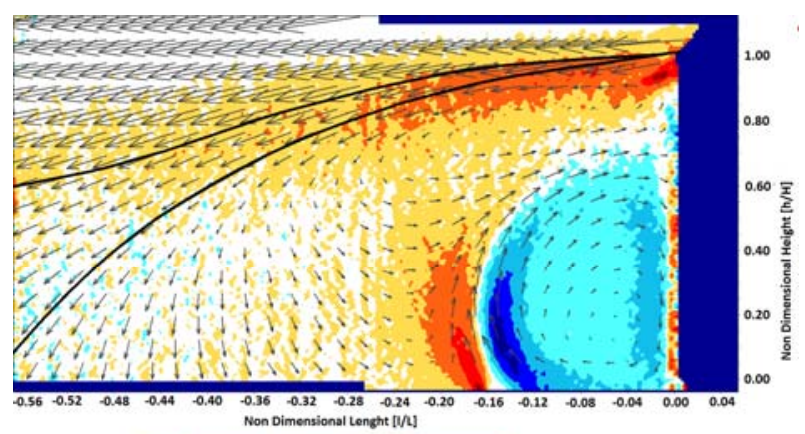

\section{TIITHIIII"}

Fig. 12 Time averaged PIV image for downward blowing configuration, $\mathrm{C} \mu=0.012 \mathrm{~h} / \mathrm{H}=10.3 \%, \mathrm{Re}=3.01 \times 10^{6}$.

It is well known that an increase in surface velocity over a flat plate will create a reduction in static surface pressure, and this theory has been used in assessing the effect on the lower vortex structure on the model base surface.

Fig. 13 shows the in-plane vertical velocity magnitudes, with and without blowing. The region covered in Fig. 13 is shown as dotted red line in Fig. 11. In the region where the lower vortex structure interacts with the model base surface, velocity magnitudes are reduced by approximately $2-4 \mathrm{~m} / \mathrm{s}$ for the blown case, relative to the un-blown case. a)
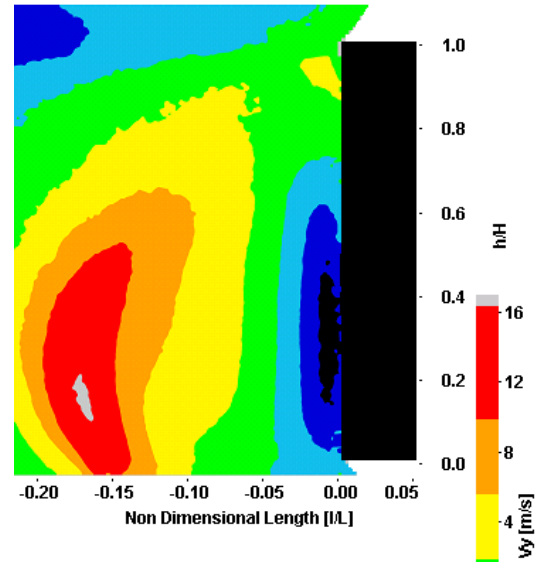

b)

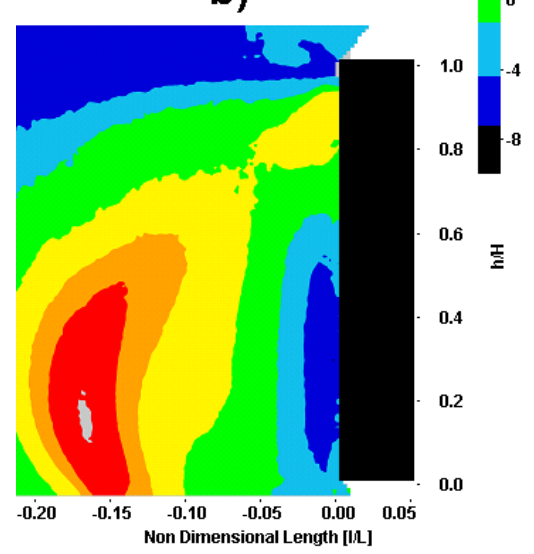

Fig. 13 Vertical velocity components for baseline (a) and blown case (b)

Littlewood (2011) investigated this effect using small trips or 'slats' placed on the base of the model to slow the surface velocities and drag reductions were achieved. In the current application the downward blowing jet reduces the rotational energy of the lower vortex structure leading to a reduction in the velocities measured at the model base surface.

\section{Net Drag Changes}

Although the drag reductions achieved are significant, the momentum coefficients required to produce the change are high and an overall gain can only be claimed once the jet power requirement is subtracted from the aerodynamic drag power saving. The net aerodynamic power saving is calculated using Equation 5 and the net power saving for Configuration $\mathrm{D}$, at various momentum coefficients, and a test Reynolds number of $3.01 \times 10^{6}$ are shown in Fig. 14.

Equation $5 A P_{N E T}=\left(\Delta F_{D} \times V\right)-\left(\dot{m}_{j} \times V_{j}^{2} \times \eta_{j}\right)$ 


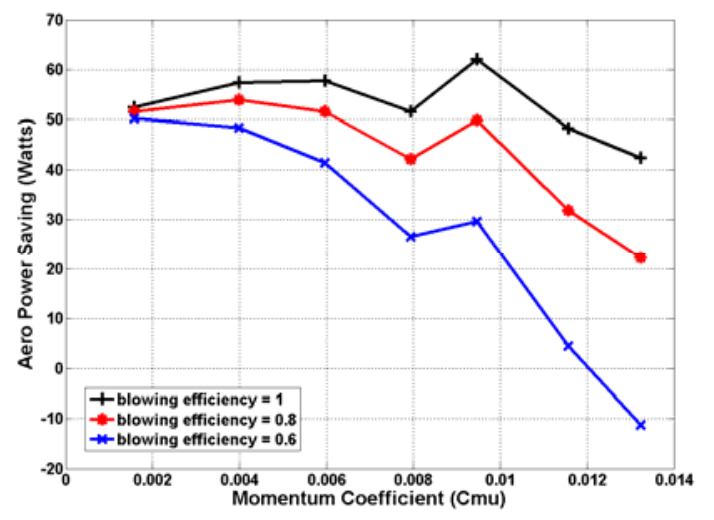

Fig. 14 Aerodynamic Power savings available at different blowing system efficiencies

If assumptions are made that the blowing system used is $100 \%$ efficient then net aerodynamic power savings are observed (Fig. 14). The savings available for the wind tunnel model tested are relatively constant up to $\mathrm{C}_{\mu}=$ 0.0095 , and range from $52.5 \mathrm{~W}$ to $62.5 \mathrm{~W}$ in this region. Above $\mathrm{C}_{\mu}=0.0095$ the aerodynamic power savings available are reduced.

If it is possible to maintain the same balance of input power to the system vs savings in aerodynamic drag power for a full scale vehicle rather than a $1 / 4$ scale model, the power savings could be up to 16 greater $(840 \mathrm{~W}$ to $1 \mathrm{~kW})$. However, it is unknown whether the same net drag power savings would be achievable because of the complexity of the scaling problem, which not only involves the scaling of the physical size of the vehicle, but also the jet dimensions, and its effect on jet performance and power consumption.

Additionally any practical system that is used to create the blowing will have losses due to efficiency, whether from compressor efficiency or losses in pipework required to install the system. The effect of this efficiency term is also included in Fig. 14, and shows a significant reduction in net power saving as $\mathrm{C}_{\mu}$ increases and $\eta_{j}$ decreases. This illustrates that although the largest $C_{d}$ reductions are achieved with the highest $\mathrm{C}_{\mu}$ available, the potential for net power savings is highest when lower $C_{\mu}$ values are used. This is substantiated further when vehicle mass increases and packaging spaces are considered; even the lower $\mathrm{C}_{\mu}$ values tested require the use of heavy and physically large compressors and storage tanks. Vehicle mass increases will create an increase in the tractive effort required to move the vehicle, and as such the smaller and lighter the blowing system can be the better.

Currently even with the potential net aerodynamic power savings demonstrated at low $\mathrm{C}_{\mu}$ values a direct application of the technology using compressors and storage tanks is unlikely to be practical due to the high mass flow rate requirements and consequently the need for large and heavy equipment. However it may be possible that similar effects may be achievable using

Page $\mid 9$ systems such as NACA ducts to passively duct freestream flow into the wake whilst maintaining the lines and appearance of a standard vehicle, and this is a suggested investigation for further work. It may also be possible to reduce the duty cycle of the jet using pulsed blowing, such that the wake flow is still modified, but the total mass flow rate requirement is reduced.

\section{CONCLUSIONS}

- The influence of steady blowing applied at a variety of angles on the roof trailing edge of a simplified $1 / 4$ scale square-back style vehicle has been investigated. Increases in measured drag coefficient were observed for all configurations tested except for the configuration D.

- The changes in drag coefficient were corrected for blockage effects and the influence of jet thrust has been subtracted. Where significant drag reductions were observed an effort has been made to understand the mechanisms which cause this change. For these configurations the majority of changes in drag forces are as a result of base pressure modification which has been supported using an area weighted base pressure coefficient calculation.

- In the cases tested at $\mathrm{Re}=3.01 \times 10^{6}$ the reduction in drag observed when blowing was applied has been attributed to a reduction in wake size via a deflection of the upper shear layer downwards and reduction in the velocities observed in the lower vortex structure located at close proximity to the base surface.

- Although the highest reductions in $\mathrm{C}_{\mathrm{d}}$ are achieved at the highest $\mathrm{C}_{\mu}$ configurations tested, an analysis of the net change in aerodynamic power has revealed that a system would be most efficient using low $\mathrm{C}_{\mu}$ configurations.

- Although the current work shows promise for the use of active blowing the current mass flow rates required would be prohibitive when trying to directly implement the techniques to a production road vehicle. The use of passive ducting of freestream flow may allow the technique to be exploited whilst still maintaining vehicle style and packaging requirements.

- $\quad$ Alternatively the mass flow rates required may be reduced by using pulsed jets to reduce the 
duty cycle of the jet whilst maintaining the wake modification behaviour.

\section{ACKNOWLEDGEMENTS}

The authors would like to thank Rob Hunter and Stacey Prentice with all their help in preparing the models for the tests carried out in this paper.

\section{REFERENCES}

Bearman P, Morel T, (1983) Effect of freestream turbulence on flow around bluff bodies. Prog. Aerospace Science Vol 20:97-123 doi:10.1016/03760421(83)90002-7

Brunn A, Nitsche W, (2006) Active control of turbulent separated flows over slanted surfaces. International Journal of Heat and Fluid Flow 27: 748-755 doi: 10.1016/j.ijheatfluidflow.2006.03.006

Englar R, (2003) Drag reduction, safety enhancement and performance improvement for heavy vehicles and SUVs using advanced pneumatic aerodynamic technology. SAE International Paper No. 2003-01-3378 doi: $10.4271 / 2003-01-3378$

Geropp D, Odenthal, H-J, (2000) Drag reduction of motor vehicles by active flow control using the coanda effect. Experiments in Fluids Vol. 28, No. 1, 74-85, doi: $10.1007 / \mathrm{s} 003480050010$

Glezer A, Amitay M, (2002) Synthetic Jets. Annual Review of Fluid Mechanics Vol. 34: 503-529. doi: 10.1146/annurev.fluid.34.090501.094913

Hollis D, (2004) Particle Image Velocimetry in Gas Turbine Combustor Flow Fields. PhD Thesis, Loughborough University

Horn H, Neubeck J, Wiedemann, J (2011) Rating Massrelated Energy Demand for Vehicles with New Powertrain Concepts. SAE International Paper No. 2011-37-0010 doi: 10.4271/2011-37-0010

Hucho W, (1998) Aerodynamics of Road Vehicles. Society of Automotive Engineers, P38

Lin J, Towfighi J, Rockwell D, (1999) Near Wake of a Circular Cylindar: Control by Steady and Unsteady Surface Injection. Journal of Fluids and Structures, vol. 9, issue 6, pp. 659-669 doi: 10.1006/jfls.1995.1036

Littlewood R, Passmore M, (2010) The Optimization of Roof Trailing Edge Geometry of a Simple Squareback. SAE International Paper No. 2010-01-0510 doi: 10.4271/2010-01-0510
Littlewood R, Passmore M, Wood D, (2011) An Investigation Into the Wake Structure of Square Back Vehicles and the Effect of Structure Modification on Resultant Vehicle Forces. SAE International Paper No. 2011-37-0015 doi: 10.4271/2011-37-0015

Pastoor M, Henning L, Noack B, King R, Tadmor G, (2008) Feedback shear layer control for bluff body drag reduction. J. Fluid Mech vol. 608:161-196 doi: $10.1017 / \mathrm{S} 0022112008002073$

Ribaldone E, (2010) Application of mulit-objective optimization to the aerodynamic development of passenger vehicles at FIAT. 8th MIRA International Vehicle Aerodynamics Conference 2010

Rouméas M, Gilliéron P, Azeddine K, (2008) Analyze and control of the near wake flow over a squareback geometry. Computers \& Fluids 38:60-70 doi:10.1016/j.compfluid.2008.01.009

Schrefl M. (2010) The Audi A1 - Aerodynamics and Aeroacoustics. 8th MIRA International Vehicle Aerodynamics Conference 2010

Schultz S. (2010) Aerodynamics of Modern Sport Utility Vehicles. 8th MIRA International Vehicle Aerodynamics Conference 2010

Sovran G. (1983) Tractive energy based formulae for the impact of aerodynamics on fuel economy over the EPA driving schedules. SAE International Paper No. 830304 doi: $10.4271 / 830304$ 